\title{
Adapting to Changes in Design Requirements Using Set-Based Design
}

\author{
Thomas A. McKenney, Lauren F. Kemink, and David J. Singer
}

\begin{abstract}
Ship design is a highly intensive and complex process mainly due to the large number of components and competing requirements. With advancement in technology, design, and evaluation processes, more emphasis has been placed on obtaining not just a feasible design, but also an optimal one. Advanced design methods such as set-based design (SBD) can provide a structured approach to evaluating the design space in order to make accurate and informed decisions toward a more globally optimal design. This paper presents the general application of the SBD process for US Naval vessels as well as a specialized focus on changes in design requirements. Specifically, the two main objectives are an evaluation of how delaying decisions using SBD could cause higher adaptability to changes later in the design process and development of a tradeoff space for evaluating reduced sets. A design experiment that simulated cycles of the SBD process was developed and implemented to provide insight into this objective. The different stages of the experiment included determining intersections between design components in the design space, narrowing variable sets to eliminate infeasible regions, and evaluating the effects of changing design requirements.
\end{abstract}

\section{Introduction}

While ship design remains a highly intensive and complex process, advanced design methods such as set-based design (SBD) can provide a structured approach to evaluating the design space while moving toward a more globally optimal design. SBD has been used for applications in the automotive and aerospace industries, but has recently been proposed for the ship design process. The main objective discussed in this paper is an evaluation of how delaying decisions using SBD could cause higher adaptability to changes later in the design process.

This paper evaluates certain aspects of SBD, not a full implementation of the SBD method for a particular design. One important aspect of SBD that is not considered is the increasing design fidelity during the design process. A design experiment that simulates rounds of the SBD process was developed to provide insight into the objective stated above. Documentation of the SBD process and information communicated during the design experiment was recorded for a clearer understanding of how SBD works.

This paper encompasses work started at the Department of Naval Architecture and Marine Engineering at the University of Michigan as a Grand Challenge project for the Office of Naval Research (ONR). The experiment was conducted at the Center for Innovation in Ship Design (CISD) at the Naval Surface Warfare 
Center-Carderock Division during the summer of 2010 .

The assigned topic for the Grand Challenge project was used as a case study for the design experiment that was conducted at CISD. The topic focuses on autonomous mine clearing and the mine countermeasure (MCM) mission. The MCM mission is completed using a vessel that deploys and recovers three types of autonomous vehicles: unmanned aerial vehicles (UAVs), unmanned surface vehicles (USVs), and unmanned underwater vehicles (UUVs). The discussed research focused on the SBD method, not the complete and proper evaluation of the MCM mission. Therefore, there are certain assumptions regarding MCM that were made in order to not detract from the main focus of the research.

\section{Introduction to SBD}

The SBD process can be defined for this experiment using the following steps:

explore the design space and develop functional groups or specialties that take part in the design process,

specialties determine the range of variables that are capable of defining each specialty's area of interest,

the variables are initially defined to allow for intersection of ranges,

through increasing levels of detail and knowledge, parts of the design space are eliminated until feasible sets remain,

these remaining sets are then limited by performance or cost metrics until a single preferred solution exists (Bernstein 1998).

Figure 1 provides a visual depiction of the SBD process. The different colored circles represent the ranges of different specialties. By exploring the design space, intersections between specialties can be identified. The black highlighted portions show these intersections. As the design progresses downwards in the figure, the sets narrow.

SBD allows engineers to evaluate tradeoffs in a conflicting design by gaining more information before making decisions. During the intersection phase of the design process, each specialty has an opportunity to influence the first set of design variables, which leads to a large set of possible solutions. The decisions are made to eliminate parts of the design space when the trade-off information is better known or eliminated by other solutions (Singer et al. 2009). At a point when all sets are feasible and all tradeoffs are explored, the best possible design is selected.

In early stage design, decisions are made that commit costs and affect performance in the final product. These decisions are made when the least amount of information is known about the design space. To delay decision making, SBD uses ranges to define variables so the design can continue until a decision is made to limit the design space. This prevents decisions being made too early based on a small amount of information. Only when sufficient knowledge of the design is known are options eliminated (Liker et al. 1996). By keeping the variables open longer, the amount of rework required is mitigated if a change is made to the design requirements.

\section{Mission}

The US Navy has conducted the MCM mission for decades, and as technologies have developed, carrying out the mission has become more effective and safer. Duties that have been completed by marine mammals and humans in the past can now be completed using advanced technologies such as autonomous vehicles.

\section{DEFINITION}

The MCM mission includes detection, classification, identification, and elimination of mines in various regions. Avoidance of mines is also considered under certain situations. The mission focus of this study is on efficient and automated mine clearing that can be conducted with efficiency and speed. Also, the mothership concept is envisioned to carry different types of autonomous vehicles.

From the general guidelines and definition of MCM, four missions were identified to cover a 
variety of possible threats. The four missions include Transiting the Sea Lines of Communication and Choke Points, Mine Avoidance and Exploiting Gaps, Battle Group Operating Area, and Port Break-In, Break-Out, and Clearance (Holder et al. 1998). Each of these missions is very different and requires the MCM ship to carry a variety of vehicles to complete the different aspects of each mission.

The four missions require different operational tasks in order to meet the objectives. These tasks include reconnaissance, search, identification, and neutralization. Reconnaissance is defined as that phase of the exploratory objective designed to make rapid assessment of limits and density of a minefield. Search is defined as the act of searching and detecting mine-like objects. Identification is defined as the act of identifying and marking mines. Neutralization is the removal of detectable mines from an assigned area. This would include the sweeping, jamming, and signature methods (Holder et al. 1998). Based on the mission definitions, not all missions require all tasks to be completed. The autonomous vehicles available to the ship complete the different tasks.

\section{AUTONOMOUS VEHICLES SELECTED}

Three types of autonomous vehicles were considered to conduct the MCM missions.

A representative list of vehicles used for MCM was generated for each type of mission. Vehicle characteristics including speed, range, and endurance were gathered to be used as part of the performance evaluation. Similar types of vehicles were identified and eliminated to simplify the design process. The final list of vehicles consisted of two UAVs, five USVs, and seven UUVs. Each vehicle can complete certain MCM tasks that are required by the four missions.

\section{INITIAL DESIGN SPACE EXPLORATION}

An initial study was completed to determine reasonable starting values for all variables and parameters used in the SBD experiment. Principal characteristics such as length, beam, draft, and displacement were considered. Also, range and speed values were compared based on ship
(1)

Design Space

(2)

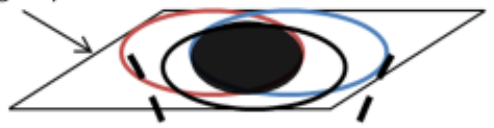

(3)

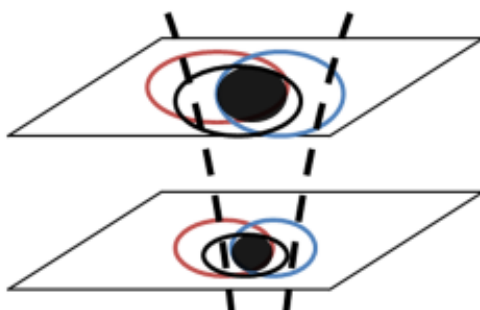

(4)
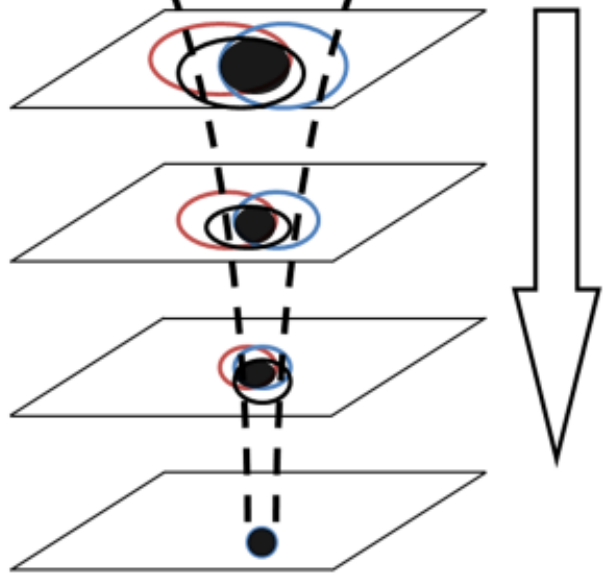

(5)

Figure 1: Set-Based Design Process (adapted from Bernstein 1998)

mission requirements. The two ships that the study used for reference were the Avenger Class MCM ship and the more recent Littoral Combat Ship (LCS).

The MCM ship conducts only MCM missions while the LCS mission modules can facilitate multiple missions. The MCM ship, although specific to the MCM mission, does not carry any unmanned vehicles. The LCS was designed to carry a variety of vehicles, including all types considered for this project. Using the research completed on these ships, and basic naval design guidelines, initial ranges of the design variables were developed.

\section{Design Experiment Preparation}

The initial stages of the SBD process require the determination of what specialties (i.e., propulsion, cost, etc.) are to be considered for the MCM vessel. Variables that are negotiated by the specialties also need to be identified. Finally, parameters that are exchanged between specialties, but do not need to be negotiated, are identified. To facilitate the SBD process, a tool and methodology is required for each specialty to complete a proper evaluation. For example, 
the resistance specialty would require a resistance prediction program and basic hull characteristics or parent hull for proper evaluation. These tools are either selected from an existing library or developed, as needed, and a methodology is created.

Another aspect of the preparation includes clearly defining the design process, including how the specialties interact and the integration required to reduce sets. In order to control the experiment, certain rules and guidelines were instituted to allow smooth transition between rounds. To facilitate proper documentation of the experiment, various spreadsheets and documents were generated to track decisions and the reasoning behind these decisions. The proper preparation discussed in this section allows the experiment to be run as smoothly as possible in order to generate valid and meaningful results.

\section{SPECIALTIES}

The specialties were selected based on general components of almost all ship design concepts as well as MCM mission specific considerations.

The generic ship design concept components, or specialties, include:

general arrangements,

weights,

resistance,

propulsion,

stability, and

cost.

Along with the generic specialties, two additional areas of interest were identified based on the MCM mission. These additional specialties include:

payload and

seakeeping.

The main function of the MCM vessel is to act as a mothership that can carry autonomous vehicles as well as launch and recover them safely. A definition of the payload, or what specific vehicles are being transported, is an important aspect of the design. The number of each vehicle and the arrangement of these vehicles within the payload spaces were used to evaluate mission performance. The critical evaluation of the payload led to the addition of the payload specialty.

Another important aspect of the mothership concept is the launch and recovery of vehicles it is carrying. The operational availability, or in what environmental conditions the vehicles can be launched and recovered, is important to consider in early stage design. The importance of ship motions and launching methods led to the addition of the seakeeping specialty. This specialty focused on reducing motions to enhance the ability to launch and recover vehicles.

\section{VARIABLES AND PARAMETERS}

Variables and parameters were selected based on their influence on the design, and if they were required by the specialties. Using the specialties identified earlier, variables were selected based on the possibility of conflicting preferences between two or more specialties. For example, the resistance specialty would prefer a smaller beam while the stability would prefer a larger beam. Variables include the principal dimensions of the ship such as length and are considered variables because the specialties have preferences for their values. The number of variables was limited in order to simplify the experiment. The nine variables chosen represent the set having the most significant impact on the design.

Parameters are information that specialties need to know, but have no specific preference for. Most parameters are passed between specialties and are based on certain inputs and outputs required by the specialties. Most of the parameters were chosen based on the type of tool the specialties used and the specific values required by the tool to run. Details on tool development are discussed in the next section.

There is also a subset of parameters that define specific requirements for the vessel. These include transit speed, transit range, and 
operational sea state. These parameters are used as inputs for some of the specialties. The experiment organizers define the minimum and maximum values for this subset of parameters. The negotiated variables and parameters can be seen in Table 1.

\section{TOOL DEVELOPMENT}

Each specialty needs a tool to complete the objective of that component of the design. These tools could range from a simple spreadsheet to sophisticated developed software. A large part of the preparation for the experiment included determining what tools should be used for each specialty. In some cases the tool had to be developed based on first principles. Also, a detailed methodology was developed to guide the person in charge of the specialty through the evaluation process. In an attempt to make the experiment run as smoothly as possible, substantial effort was put into making sure the specialty evaluation process was as clear and user-friendly as possible.

After defining the tools used by the specialties, the inputs and outputs can be identified to form a better idea of how the variables and parameters interact with the specialties. Selecting the specialty tools also dictate certain parameters that were required. Table 1 provides the

\section{TABLE 1: List of Negotiated Variables, Parameters, Requirement Ranges, and Interactions}

\begin{tabular}{|c|c|c|c|c|c|c|c|c|c|}
\hline & Unit & Resistance & Propulsion & Stability & Arrangement & Weight & Seakeeping & Cost & Payload \\
\hline \multicolumn{10}{|l|}{ Negotiated variable } \\
\hline Length & $\mathrm{m}$ & $\mathrm{N}$ & & $\mathrm{N}$ & $\mathrm{N}$ & $\mathrm{N}$ & $\mathrm{N}$ & $\mathrm{N}$ & \\
\hline Beam & $\mathrm{m}$ & $\mathrm{N}$ & & $\mathrm{N}$ & $\mathrm{N}$ & $\mathrm{N}$ & $\mathrm{N}$ & $\mathrm{N}$ & \\
\hline Depth & $\mathrm{m}$ & & $\mathrm{N}$ & $\mathrm{N}$ & $\mathrm{N}$ & In & & $\mathrm{N}$ & \\
\hline Draft & $\mathrm{m}$ & $\mathrm{N}$ & $\mathrm{N}$ & $\mathrm{N}$ & & $\mathrm{N}$ & $\mathrm{N}$ & $\mathrm{N}$ & \\
\hline USV/UUV area & $\mathrm{m}^{2}$ & & & & $\mathrm{~N}$ & & & & $\mathrm{~N}$ \\
\hline UAV area & $\mathrm{m}^{2}$ & & & & $\mathrm{~N}$ & & & & $\mathrm{~N}$ \\
\hline Engine room length & $\mathrm{m}$ & & $\mathrm{N}$ & & $\mathrm{N}$ & & & & \\
\hline Block coefficient $\left(C_{B}\right)$ & & $\mathrm{N}$ & & In & & & $\mathrm{N}$ & In & \\
\hline VCG & $\mathrm{m}$ & & & $\mathrm{N}$ & In & & $\mathrm{N}$ & & \\
\hline Length of USV/UUV cargo & $\mathrm{m}$ & & & & $\mathrm{N}$ & & & & 0 \\
\hline Length of UAV cargo & $\mathrm{m}$ & & & & $\mathrm{N}$ & & & & 0 \\
\hline \multicolumn{10}{|l|}{ Requirement ranges } \\
\hline Transit speed & kts & $\ln$ & & & & $\ln$ & & & \\
\hline Transit range & $\mathrm{nm}$ & & & & & In & & & \\
\hline Complement & & & & & In & & & & \\
\hline \multicolumn{10}{|l|}{ Parameter ranges } \\
\hline Required thrust & $\mathrm{N}$ & 0 & $\ln$ & & & & & & \\
\hline Propeller diameter & $\mathrm{m}$ & & In & & & & & & \\
\hline \# Superstructure decks & & In & & & 0 & & & & \\
\hline Transit power & $\mathrm{kW}$ & & 0 & & & In & & In & \\
\hline Structural weight & $\mathrm{kg}$ & & & & & 0 & & $\ln$ & \\
\hline Outfit weight & $\mathrm{kg}$ & & & & & 0 & & $\ln$ & \\
\hline Propeller RPMs & & & 0 & & & & & $\ln$ & \\
\hline Displacement & $\mathrm{mt}$ & & & & & 0 & & $\ln$ & \\
\hline Sea state & & & & & & & 0 & & \\
\hline Engine SFC & $\mathrm{kg} / \mathrm{kw}-\mathrm{hr}$ & & & & $\ln$ & & & & \\
\hline Prismatic coefficient & & In & & & & & & & \\
\hline Midship coefficient & & $\ln$ & & & & & & & \\
\hline Waterplane coefficient & & In & & & & & & & \\
\hline Wake fraction & & 0 & In & & & & & & \\
\hline UAV weight & $\mathrm{kg}$ & & & & & & & & 0 \\
\hline USV/UUV weight & $\mathrm{kg}$ & & & & & & & & 0 \\
\hline
\end{tabular}

In. input; O, output; N, negotiated; UAV, unmanned aerial vehicle; USV, unmanned surface vehicle; UUV, unmanned underwater vehicle. 
interactions between the variables and parameters with the specialties. This gives an overview of the inputs and outputs of each specialty as well as a look at what variables and parameters are important to the specialties.

\section{EXPERIMENT DESIGN PROCESS}

The design process for the experiment differs in certain aspects from the typical SBD method. It is important to note that the scope on this research is limited and the main focus was to evaluate how SBD handles changes in requirements. To simplify the problem, assumptions were made in regards to specialty interactions and the integration process.

One of the simplifications made for the experiment was related to how the specialties interact. If fully implementing the SBD approach, some level of communication would occur between specialties directly. It is important that these communications are documented very closely. To simplify this component of the process, the specialties only interacted directly with the integration team.

Figure 2 shows a visual representation of the interactions between the specialties. It can be seen that all specialties only communicate with the integration team, which has the central role of gathering and distributing information. The lines pointing into the center represent the information being received by the integration team and the lines pointing out of the center represent the distribution of information from the integration team to the specialties.

Figure 3 shows how the process works. Initially, a range of parameters and variables are defined by the integration team based on the initial design space exploration discussed earlier. These ranges are then distributed to the specialties. The specialties take these values and use a tool to evaluate their component of the design. They take the results of their evaluation and provide preferences for certain values of sections of the ranges to the integration team. Preferences are provided using two different methods.

The first is a preference curve, which is a graph that provides preferences for specific values in a variable range by giving a rating between zero and one. A zero rating would be that the value is infeasible. A one rating would be that it is the best, or one of the best, values. The second is information that cannot be captured in a preference curve. Any type of recommendations or qualitative information that the specialty wants to be known is transferred to the integration team.
Figure 2: Interactions Between the Specialties in the Design Experiment

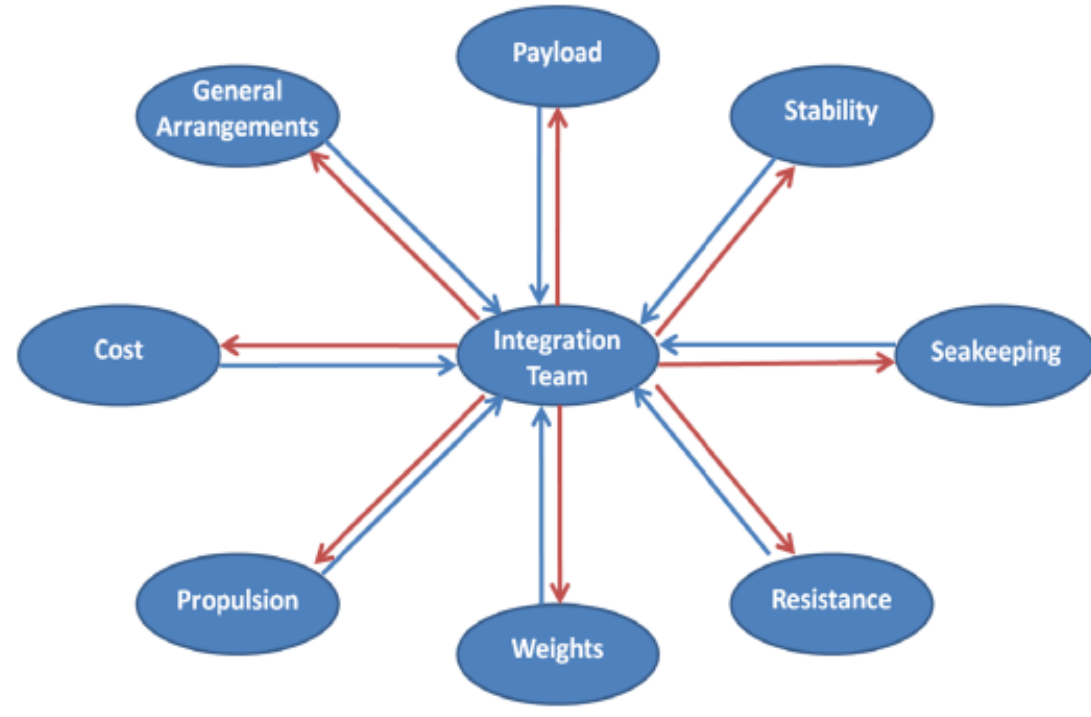




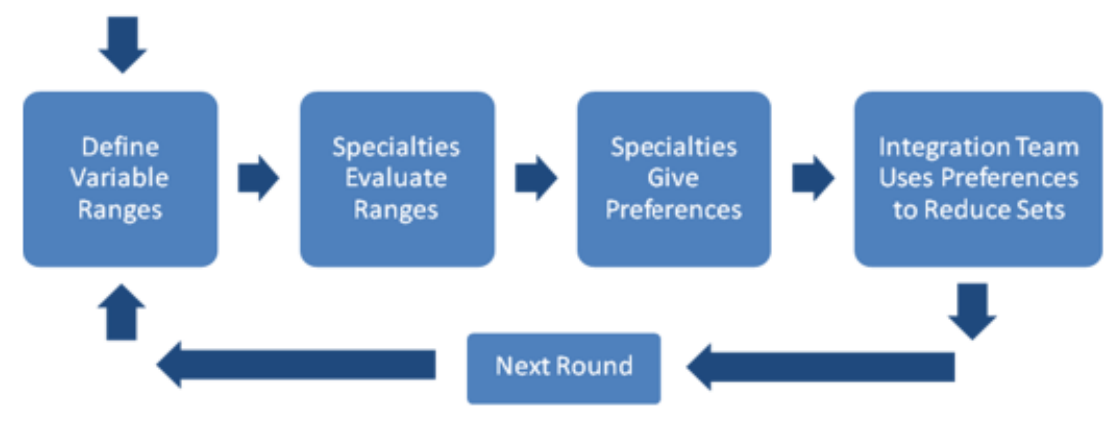

The integration team then uses the preferences of the specialties to determine a reduced set. The preference curves are combined to form a single combined preference curve. All other information gathered is considered and the integration team makes a decision to reduce the range for all the variables. The next round of the process begins when the integration team distributes out reduced ranges for the variables.

The integration team has a very important role in the process because all major decisions are decided at this level. The process begins by gathering all the preference information provided by the specialties, which includes preference curves and their comments and recommendations. A combined preference curve is then formed that includes all the specialty preferences for each variable. Throughout the whole process it is important to record and document all factors involved in the design and the decision-making process. Next, each variable is evaluated individually and a reduced set is chosen. The final stage is taking the reduced sets and distributing the updated information to the specialties for the next round.

\section{DOCUMENTATION}

Various techniques were used throughout the experiment to properly document the process. Along with the actual preference curve for each variable, the specialties had to comment on the reasoning behind the shape of the preference curve. These comments are used by the integration team to make decisions and are then recorded. The integration team also records decision-making reasoning as well as what the major impacts were for the design. It is important to note that not all preferences are equal. If one specialty has a major driver, other preferences are not considered as important when making decisions.

\section{SBD Experiment}

The SBD experiment was designed and implemented based on the objectives outlined earlier in this paper. The experiment was a simulation of the process, not a complete implementation. A total of 10 volunteers were used in the experiment and assigned to the specialties outlined earlier. Some specialties required more timeconsuming tasks; therefore, more than one person was assigned to some of the specialties.

The experiment was completed over the course of 4 days. The first day focused on training the volunteers on the tools used for the specialties, allowing individuals to become familiar with the tools and methodologies provided and ask clarifying questions. The first official round took place the second day and focused on determining intersections between specialties in the design space. It is important to first evaluate the design space and determine whether there are intersecting points between specialties. If some specialties do not intersect, some of the ranges must be expanded to create these intersections.

The second round focused on narrowing the variable sets to eliminate infeasible regions. If specific values are not feasible for any specialty, they are eliminated completely from the 
Figure 4: Determining Intersection Between

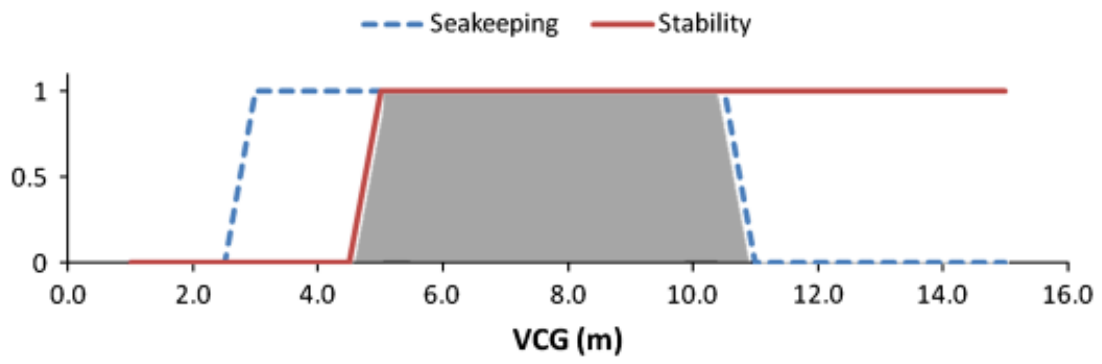

evaluation. Round 3 focuses on evaluating the effects of changing design requirements. This round was used to form some conclusions based on our research objectives.

\section{DETERMINING INTERSECTIONS}

The first round focused on determining intersections between specialties. Intersections can be determined using the variable preferences provided by the specialties. Figure 4 shows an example of an intersection between two specialties. This graph shows two different preference curves for the VCG. The blue hashed line is the seakeeping preference and the red solid line is the stability preference. The gray shaded area shows the intersection between the specialties.

It might seem interesting that stability has favorable preferences for higher VCG values than seakeeping. The main reason this is occurring is because of the guidelines used in determining preferences. If a value is feasible for at least one design within the ranges, it remains part of the set. For this specific preference, stability shows that the higher values for VCG could work for some of the larger ships even if it might fail with the smaller ships.

In some cases the initial ranges are not completely accurate and this can be seen in the specialty preferences. As mentioned earlier, the initial ranges were determined based on previous knowledge of vessels and engineering judgment. The first round was also used to determine whether initial ranges for all the variables and parameters were reasonable. Most sets were reduced or stayed the same, which shows that the associated variable and parameter ranges were reasonable. A typical convergence can be seen in
Figure 5. For the beam, it can be seen that the upper and lower bounds either remain the same or are reduced.

If the initial ranges are not adequate, the specialty preferences will show that they wish to explore beyond the given range. A good example of this is the Mission Bay Length variable.

Figure 6 shows the first round preference for the Mission Bay Length.

It can be seen that values do not start to be feasible until closer to the upper bound. The integration team took this preference under consideration and then expanded the upper bound to allow for further exploration. The convergence diagram for the Mission Bay Length is provided in Figure $\mathbf{7}$.

The initial ranges for the variable and parameters, although important, can be verified during the first round through the evaluation of specialty preferences. This leads to a proper and complete exploration of the design space and the required information to not only show where the design should be heading, but also where the design should not go and why. After all intersections

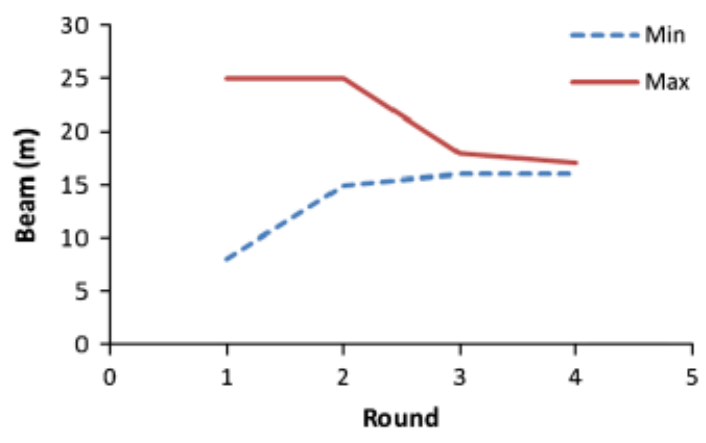

Figure 5: Example Convergence Diagram 
between specialties are identified, the sets can be reduced based on these intersections.

\section{ELIMINATE INFEASIBLE REGIONS}

After looking for intersections between specialties, the infeasible regions can be identified through specialty preferences. The specialty preference curves are combined and the infeasible regions are identified by zero values in the preference curves. The set can then be reduced by eliminating the infeasible regions.

If any of the preference curves has a zero for a specific value, it remains a zero in the combined preference curve. This is because if it is not feasible for one preference, then it is not feasible for the whole design. Once the preference curves are combined, the integration team can reduce the set based on feasibility. The reduced set will then be given to the specialties to evaluate in the next round.

\section{CHANGING REQUIREMENTS}

One of the main focuses of the research was to assess how SBD can handle changing requirements later in the design process. Delaying decisions using SBD was also an important component. As mentioned earlier, the experiment is attempting to simulate the SBD process. One aspect of simulating delaying decisions was using sets for the speed and range requirements for the ship. In most designs, a specific value for speed and range is chosen at the beginning of the process. As the rounds progressed, the sets were narrowed similarly to the variable sets to show how decisions like these can be delayed until further in the process.

Round 2 also focused on how changing different types of requirements would affect the SBD process. Two changes in requirements were made before Round 3. These included an addition of storage and flight deck space for one $\mathrm{MH}-60 \mathrm{~S}$ helicopter and the addition of a $57 \mathrm{~mm}$ deck gun and ammunition stores in the bow. These changes affected the specialty preferences for certain dimensions from Round 2 to Round 3. Figures 8 and $\mathbf{9}$ show the preference curves for

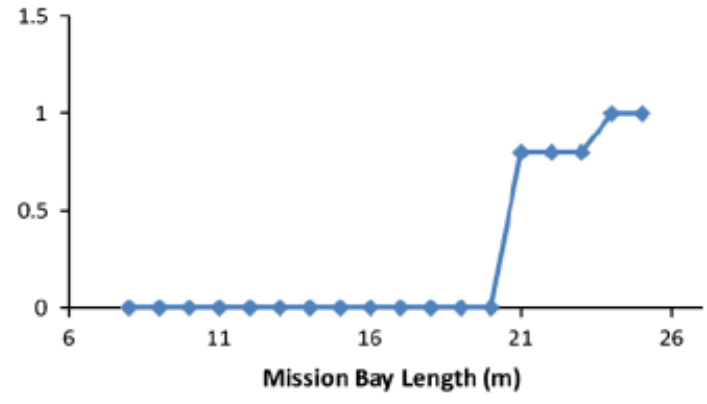

Figure 6: Mission Bay Length Preference Curve (Round 1)

the Hangar Length after Round 2 and Round 3. It can be seen that in Round 2 the preference remained the same after $16 \mathrm{~m}$. After the introduction to the requirement changes, the preference changed to favor the upper portion of the set.

As a result of the SBD process, the impact of the requirement changes could be seen through the preferences of the specialties. The sets were also open enough to accommodate these changes within the open sets. It is important to note that these changes only affected a small number of specialties and, overall, did not have a major impact on the design. In further work, the magnitude of the requirement changes could vary to determine how robust the SBD process is. Even using these smaller scale changes, the most important aspect of SBD captured in this experiment is being able to see how the requirement changes affect the design.

\section{EXPERIMENT CONCLUSIONS}

The final results of the SBD experiment were in a form of reduced sets for all the variables that the

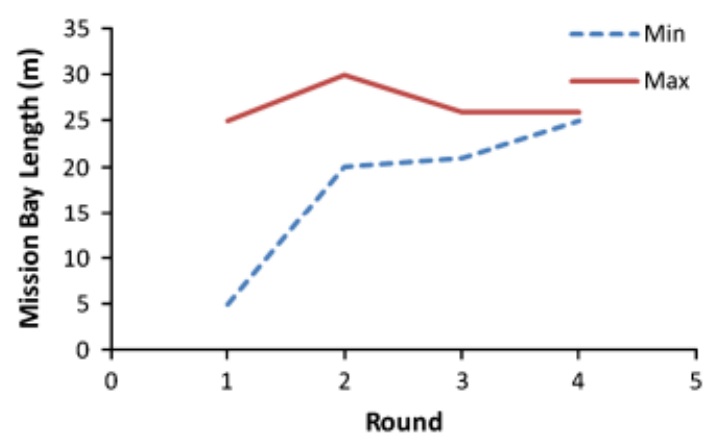

Figure 7: Mission Bay Convergence Diagram 
Figure 8: Hangar Curve (Round 2) Length Preference
Figure 9: Hangar Length Preference Curve (Round 3)

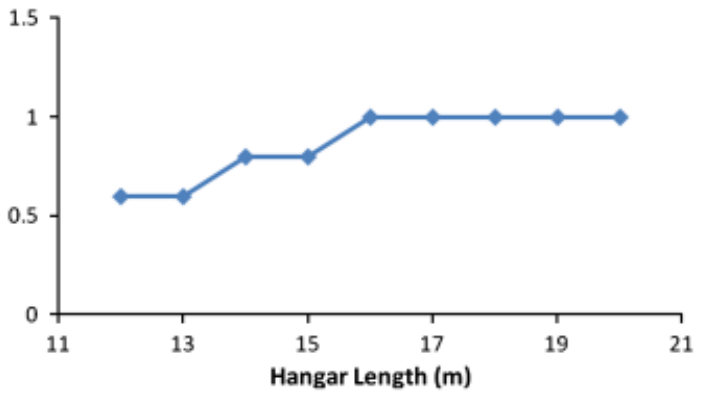

specialties negotiated. The experiment did not produce a single design or a series of designs. Starting with the initial ranges, each set was reduced dramatically to a much more reasonable and manageable range. Table 2 provides the final design characteristics from the design experiment. For example, the original length range spanned $100 \mathrm{~m}$. The final length range was $5 \mathrm{~m}$.

There are three major conclusions that can be drawn from the SBD experiment. These are:

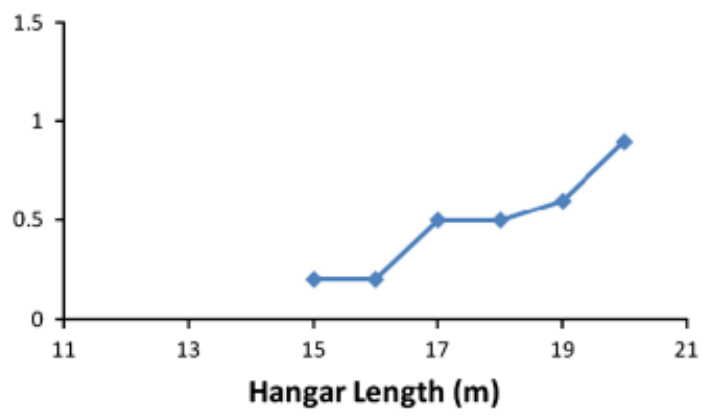

TABLE 2: Final Design Characteristics

\begin{tabular}{lcccccc} 
& \multicolumn{2}{c}{ Initial Ranges } & & \multicolumn{2}{c}{ Final Ranges } \\
\cline { 2 - 3 } \cline { 6 - 6 } & Minimum & Maximum & & Minimum & Maximum \\
\hline Length $(\mathrm{m})$ & 50 & 150 & & 95 & 100 \\
\hline Beam $(\mathrm{m})$ & 8 & 25 & & 16 & 17 \\
\hline Depth $(\mathrm{m})$ & 5 & 15 & & 9 & 11 \\
\hline Draft $(\mathrm{m})$ & 2.5 & 5 & & 5 & 6 \\
\hline Block coefficient & 0.4 & 0.7 & & 0.5 & 0.6 \\
\hline VCG $(\mathrm{m})$ & 1 & 15 & & 5.8 & 6.5 \\
\hline Engine room length $(\mathrm{m})$ & 15 & 40 & & 15 & 23 \\
\hline Mission Bay Length $(\mathrm{m})$ & 5 & 25 & & 25 & 26 \\
\hline Hanger length $(\mathrm{m})$ & 4 & 20 & & 17 & 19 \\
\hline
\end{tabular}

- Unrealistic initial ranges can be corrected based on specialty preferences.

- Changes in requirements can be handled by the SBD method, due to the robustness of the process:

Specific values such as speed and range do not have to be chosen at the beginning.

Variable and parameter ranges were open enough to allow for changes.

- The SBD method allows you to see how changes impact the design.

The scope of the project and experiment was narrow and did not cover all aspects of SBD, but the goal of the experiment was achieved. The evaluation of delaying decisions using SBD and how requirement changes can be handled are seen through the experiment results. In order to thoroughly evaluate the handling of requirement changes, additional experiments would need to be conducted. As mentioned earlier, implementing various magnitudes of change would help form a better conclusion on how robust the SBD process really is. Regardless of the magnitude of requirement change, the SBD process allows you to see the impacts that the changes have on the design.

\section{References}

Bernstein, J. I., "Design methods in the aerospace industry: looking for evidence of set-based practices." Master of Science thesis, Massachusetts Institute of Technology, 1998.

Holder, G.S. and J.E. Rhodes, Marine Corp report: "Concept for Future Naval Mine Countermeasures in Littoral Power Projection." May 1, 1998. Available at: https://www.mccdc.usmc.mil/futures/concepts/ mcm.pdf.

Liker, J.K., D.K. Sobek II., A.C. Ward, and J.J. Cristiano, "Involving suppliers in product development in the United States and Japan: evidence for set-based concurrent enģineering," IEEE Transactions on Enģineering Manaǵement, Vol. 43, No. 2, pp. 165-178, May 1996.

Singer, D.J., "A hybrid agent approach for set-based conceptual design through the use of a fuzzy logic agent to facilitate communications and negotiation." Ph.D. dissertation, University of Michigan, 2003. 
Singer, D.J., N. Doerry, and M.E. Buckley, "What is setbased design?," Naval Engineering Journal, Vol. 121, No. 4, pp. 31-43, October 2009.

"The Navy Unmanned Surface Vehicle (USV) Master Plan." July 23, 2007.

\section{Huthor Biographies}

Thomas A. McKenney is a Ph.D. student in the Department of Naval Architecture and Marine Engineering at the University of Michigan. He is concurrently earning a M.S.E. in Industrial and Operations Engineering. He earned a B.S.E. in NA\&ME from the University of Michigan in 2009. His research interests include early-stage design and design optimization.

Lauren F. Kemink is a Graduate Student, University of Michigan, Department of Naval Architecture \& Marine Engineering.

David J. Singer is an Assistant Professor and CoDirector of the Naval Engineering Education Center (NEEC) in the Department of Naval Architecture and Marine Engineering (NAME) at the University of Michigan College of Engineering. Dr. Singer's research focuses on ship production, ship design, and systems integration. His research on Set-Based Design led to his 2007 ONR Young Investigator Award. Dr. Singer obtained a B.S.E. degree in NAME, M.Eng. degree in Concurrent Marine Design, M.S.E. degree in Industrial and Operations Engineering and a Ph.D. in NAME all from the University of Michigan. 